\title{
Letter to the Editor concerning "Can standard anterior Smith-Robinson supramanubrial approach be utilized for approach down to T2 or T3?" by Singhatanadgige W, Zebala LP, Luksanapruksa P, Riew KD [Eur Spine J (2017) 26:2357-2362]
}

\author{
Vishal Kumar $^{1} \cdot$ Udai Cheema ${ }^{1} \cdot$ Prasoon Kumar $^{1}$ \\ Received: 13 November 2017 / Accepted: 5 December 2017 / Published online: 13 December 2017 \\ (c) Springer-Verlag GmbH Germany, part of Springer Nature 2017
}

\section{Dear Editor,}

We came across and read with great interest the article entitled "Can standard anterior Smith-Robinson supramanubrial approach be utilized for approach down to T2 or T3?" published in the European Spine Journal [Singhatanadgige W, Zebala LP, Luksanapruksa P, Daniel Riew K (2017) Can standard anterior Smith-Robinson supramanubrial approach be utilized for approach down to T2 or T3? Eur Spine J; 26:2357-2362].

We commend the authors for establishing this novel technique by which one can decide the approach to the lower cervical and upper thoracic spine. However, we would like to raise a few pertinent points, where we highlight a few limitations in the study.

First of all, regarding the technique, the authors mention the usage of simple lateral view of cervical spine and drawing a straight line from the proposed skin incision to the lowest instrument level.

However, a simple lateral view often does not delineate all seven cervical vertebrae and is often insufficient to visualize the cervico-thoracic junction. A swimmer's view is often needed for the latter and that too is not sufficient in every case [Rethnam U, Yesupalan RS, Bastawrous SS (2008) The Swimmer's view: does it really show what it is supposed to show? A retrospective study. BMC Med Imaging; 8:2]. Therefore, visualization further down to the level of the first-to-third thoracic vertebrae is very unlikely in majority of cases, unless the patient has a long neck. In a short-statured patient with a short neck, we believe that this methodology might not work. Therefore, how the authors were able to base the study solely on a simple lateral view requires some explanation.

Second, in cases of trauma, where the vertebral bodies could be deformed or collapsed, drawing a straight line as suggested in the article could be a difficult proposition.

Third, the authors themselves agree that anterior approach to visualize cervico-thoracic junction is tedious, limited, and technically demanding. In addition, we believe that intraoperatively, visualizing the upper dorsal region under the $\mathrm{C}$-arm with patient supine, itself is very difficult and often impossible. Therefore, one can never be $100 \%$ sure about the level that he is working at, during the surgery. Therefore, the feasibility of anterior approach for levels between $\mathrm{T} 1$ and $\mathrm{T} 3$ vertebrae is not apt in all the cases and will require a surgeon of highest skills. This has a very high and steep learning curve for sure.

We would again like to point that the idea is a novel one, but for a wide implementation in our day-to-day practice, some loopholes need to be rectified.

\section{Compliance with ethical standards}

Conflict of interest The authors declare that they have no competing interests.

Prasoon Kumar

drprasoonksingh@gmail.com

Vishal Kumar

drkumarvishal@gmail.com

1 Department of Orthopaedics, PGIMER, Sector-12,

Chandigarh, India 